\title{
Robust state preparation of a single trapped ion by adiabatic passage
}

\author{
Chr. Wunderlich, Th. Hannemann \\ National University of Ireland, Maynooth, Co. Kildare, Ireland * \\ T. Körber, H. Häffner, Ch. Roos, W. Hänsel, R. Blatt \\ Institut für Experimentalphysik, Universität Innsbruck, Technikerstrasse 25, A-6020 Innsbruck, Austria \\ F. Schmidt-Kaler \\ Institut für Quanteninformationsverarbeitung, Universität Ulm, Einstein 11, D-89069 Ulm, Germany
}

(Dated: October 15, 2018)

\begin{abstract}
We report adiabatic passage experiments with a single trapped ${ }^{40} \mathrm{Ca}^{+}$ion. By applying a frequency chirped laser pulse with a Gaussian amplitude envelope we reach a transfer efficiency of $0.990(10)$ on an optical transition from the electronic ground state $\mathrm{S}_{1 / 2}$ to the metastable state $\mathrm{D}_{5 / 2}$. This transfer method is shown to be insensitive to the accurate setting of laser parameters, and therefore is suitable as a robust tool for ion based quantum computing.
\end{abstract}

PACS numbers: 03.67.Lx,32.80.Qk

It is the interplay between different technologies that is stimulating novel developments aiming at the ambitious goal of a future large-scale quantum computer [1]. As recent research has shown, considerable promise lies in the application of nuclear magnetic resonance (NMR) technology to ion-trap based quantum computing 2, 3]. While ion based quantum computing has strong assets concerning the preparation of multi-particle entangled states [4, 5] and the highly efficient readout of qubit states using projective measurements [6, 7, [8], liquid state NMR quantum computing relies on well developed radio frequency (rf) techniques which have enabled the most complex [9, 10, 11] sequences of quantum logic gate operations to date with about $10^{2}$ to $10^{3}$ rf-pulses [12]

The basic construction principle of an elementary quantum computer with trapped ions relies on linear cold ion crystals serving as quantum register. Two of each of the ions' electronic states serve to store elementary bits of quantum information (qubits) which are coherently manipulated by the application of laser 13 or microwave pulses [14] with well defined timing, frequency and phase. With a number of operations applied, on single ions individually or on groups of ions a quantum algorithm may be implemented.

Composite gate operations 2], initially developed in the context of NMR experiments, have already enabled complex tasks in ion traps like the demonstration of quantum teleportation [6, 7], which comprises about 30 laser pulses of different frequency, phase and amplitude. In order to further increase the complexity of algorithms and to improve the robustness of single and multiqubit quantum logic gates, all parameters characterizing the

*Present address: University of Siegen, Fachbereich Physik, 57068Siegen, Germany electromagnetic field driving qubit transitions have to be freely adjustable, thus allowing for the implementation of pulses with arbitrary amplitude and phase envelope. For this purpose, a suitable waveform having these characteristics is digitally generated in the rf-domain and then mapped phase-coherently onto a fixed frequency laser or microwave field for qubit manipulation. Here, as a first application we demonstrate robust adiabatic passage (RAP) in a single trapped ion qubit system.

In this publication first we briefly review some elements of the theory of rapid adiabatic passage (RAP), then give a short description of the experimental setup which allows the generation of complex laser pulses. Subsequently, experimental data demonstarting RAP are compared to the expected outcome. Finally, we sketch a number of possible applications of the RAP method as elements of a toolbox for ion based quantum computing.

For the theoretical description of RAP we model the atom as a two-level system with quantum states $|0\rangle$ and $|1\rangle$ and use a frequency-chirped laser light field coupling both levels. The interaction sweeps through the resonance $\nu_{\text {atom }}=\omega_{\text {atom }} /(2 \pi)$ of the two levels. It is convenient to define a detuning rate $\Delta \nu / T$, where $\Delta \nu$ is the frequency difference of start and stop frequency of the field driving the atomic transition and $T$ the time duration of the chirp. During the chirp, the light field amplitude is smoothly turned on and off with a Gaussian envelope, proportional to $\exp \left(-(t-T / 2)^{2} /\left(2 \sigma^{2}\right)\right)$ with $\sigma=T /(6 \sqrt{2})$ and truncated at times $t=0$ and $t=T$, see Fig. 1(a) and (b). The maximum of the light field amplitude is reached at the frequency $\nu_{\text {atom }}$. The resulting atomic 2-level dynamics is easily modeled using optical Bloch equations. A qualitative and intuitive picture can be derived if we consider a Bloch sphere representation of our system. Here the state vector

$$
\psi=c_{0}|0\rangle+c_{1}|1\rangle
$$


is written as a vector $\mathbf{R}$ with components $R_{x}=c_{0} c_{1}^{*}+$ $c_{0}^{*} c_{1}, R_{y}=i\left(c_{0} c_{1}^{*}-c_{0}^{*} c_{1}\right), R_{z}=\left|c_{0}\right|^{2}-\left|c_{1}\right|^{2}$. Likewise, an interaction $\Omega$ is written as vector $\boldsymbol{\Omega}=\left(\operatorname{Re}\left(H_{01}\right)\right.$, $\left.\operatorname{Im}\left(H_{01}\right), \delta\right)$, where $H_{01}$ is the interaction Hamiltonian (in a frame rotating with angular frequency $\omega_{\text {atom }}$ ) where $\hbar \omega_{\text {atom }}$ denotes the energy separation between levels $|0\rangle$ and $|1\rangle$ and $\delta$ the detuning of the driving field from the atomic resonance. The long lifetime of both atomic levels and the fast dynamics allows us to neglect spontaneous decay and dephasing [15]. Under these conditions the equation of motion for the Bloch vector dynamics can then be formulated [16] simply as $\mathbf{R}(t)=\boldsymbol{\Omega}(t) \times \mathbf{R}(t)$, in obvious analogy with magnetic resonance phenomena. First demonstrations of RAP in ensembles have been in magnetic resonance phenomena 17], and in a variety of optical applications, see [18, 19, 20] and also references therein.

If we start off with a weak electro-magnetic field tuned below resonance and with the atom in $|0\rangle, \Omega$ will be almost aligned with the Bloch vector at the south pole of the sphere. Because both vectors point nearly in the same direction, the resulting Bloch nutations are of small amplitude. By increasing the frequency of the driving field towards resonance, thus reducing $\delta$, and simultaneously increasing the interaction strength, $\boldsymbol{\Omega}$ moves toward the equator of the Bloch sphere. If this is done slowly enough the Bloch vector $\mathbf{R}$ adiabatically follows $\boldsymbol{\Omega}$. At the equator we start to lower the interaction strength again and continue to increase the driving frequency, until $\boldsymbol{\Omega}$ and the Bloch vector point toward the north pole: adiabatic transfer has taken place. The corresponding trajectory of the Bloch vector during RAP is numerically evaluated and shown in Fig. 1c). As long the adiabaticity condition is fulfilled, with a temporal change $|\dot{\boldsymbol{\Omega}}| /|\boldsymbol{\Omega}| \ll|\boldsymbol{\Omega}|$, the transfer is robust and can be achieved over a broad range of parameters. The limits of adiabaticity are illustrated by a second Bloch nutation, with the passage through resonance scanned too rapidly (i.e., $\Delta \nu / T$ is too large), see Fig. 1(d). Residual nutations about the state $|1\rangle$ are clearly visible and the final population of state $|1\rangle$ at the end of the laser pulse depends strongly on the exact duration and intensity of the pulse.

For the experiments, a single ${ }^{40} \mathrm{Ca}^{+}$ion is stored in the effective harmonic potential of a linear Paul trap. For a detailed description of the experimental setup we refer the reader to 21]. Under typical operating conditions we observe axial and radial motional frequencies $\left(\omega_{\mathrm{ax}}, \omega_{\mathrm{rad}}\right)=2 \pi(1.2,5.0) \mathrm{MHz}$, respectively. The ion is Doppler-cooled on the $\mathrm{S}_{1 / 2}$ to $\mathrm{P}_{1 / 2}$ transition near $397 \mathrm{~nm}$ to a mean phonon number of $\left(\bar{n}_{\text {radial }}, \bar{n}_{\text {axial }}\right)=$ $(5,15)$. The electronic level $\mathrm{S}_{1 / 2}, m_{j}=-1 / 2$ is identified with $|0\rangle$ and $\mathrm{D}_{5 / 2}, m_{j}=-1 / 2$ with $|1\rangle$, respectively. For the coherent manipulation on this transition, we modulate the output of a Ti:Sapphire laser with an acoustooptical modulator $(\mathrm{AOM})$ in double-pass configuration. The radio frequencies and phases that are applied to the

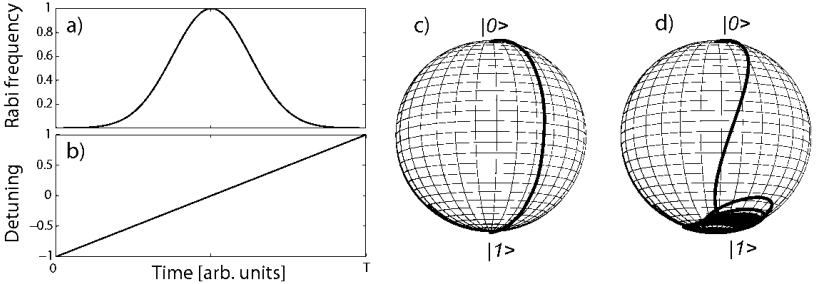

FIG. 1: Sketch of the temporal variation of the rf-waveform to form the laser pulse used for the experiments. The amplitude envelope of the light field is proportional to the Rabi frequency and hs a Gaussian shape. The envelope is plotted in units of the maximal Rabi frequency obtained during the laser pulse (a). The linear frequency chirp (b) is plotted units of $\Delta \nu$, the frequency range over which the detuning $\delta$ is swept during an individual laser pulse. For the experimental studies, we vary the pulses by variation of the peak laser intensity, thus changing the Rabi frequency, and by variation of $\Delta \nu$, the chosen frequency range of the detuning. c) Pictorial illustration of the RAP method using the Bloch sphere representation of the atomic two-level system. With $\Delta \nu=400 \mathrm{kHz}$ the evolution Bloch vector evolution is fully adiabatic and results in perfect transfer. d) Same as c), however with $\Delta \nu=1400 \mathrm{kHz}$. Here, the population transfer fails to be adiabatic. The parameters used for the simulations shown in c) and d) are identical to those in the experimental situation of fig. 2.

AOM transfer directly to the light field 28].

The complex rf-waveform is provided by a novel versatile frequency generator (VFG) that is based on a fieldprogrammable gate array. Important specifications are: a frequency range from 0 to $150 \mathrm{MHz}$, an amplitude resolution of 16 bit, the option to lock to an external clock (in our case a Rb-atomic clock), a switching time of $5 \mathrm{ns,}$ phase continuous or phase coherent switching between arbitrarily many frequencies, multiple frequencies output, and an interface that is programmable via a USB bus using a personal computer. Without the typical restrictions due to a limited memory, in this device the amplitude, phase and frequency of arbitrarily many shaped rf-pulses are freely programmable.

To match the required AOM frequency near $230 \mathrm{MHz}$, we mixed the output of the VFG near $80 \mathrm{MHz}$ with the $150 \mathrm{MHz}$ signal from an rf-synthesizer [29]. Unwanted frequency components (e.g. near $70 \mathrm{MHz}$ ) from the mixer are rejected by the AOM [30] due to its limited bandwidth.

In the experiment, for a demonstration of RAP transfer, we apply a temporal sequence to a single ion: a) The ion is prepared initially in $|0\rangle, \mathrm{b}$ ) a RAP transfer pulse $|0\rangle \rightarrow|1\rangle$ is applied and c) the excited state population $P_{|1\rangle}$ is detected by an electron shelving technique 24]. This is accomplished by driving the $S_{1 / 2}-P_{1 / 2}$ and $D_{3 / 2}-P_{1 / 2}$ dipole transitions with laser light near 397 and $866 \mathrm{~nm}$ and monitoring the blue fluorescence emitted by the ion with a photomultiplier. The internal state of the ion is discriminated with an efficiency close to $100 \%$. 


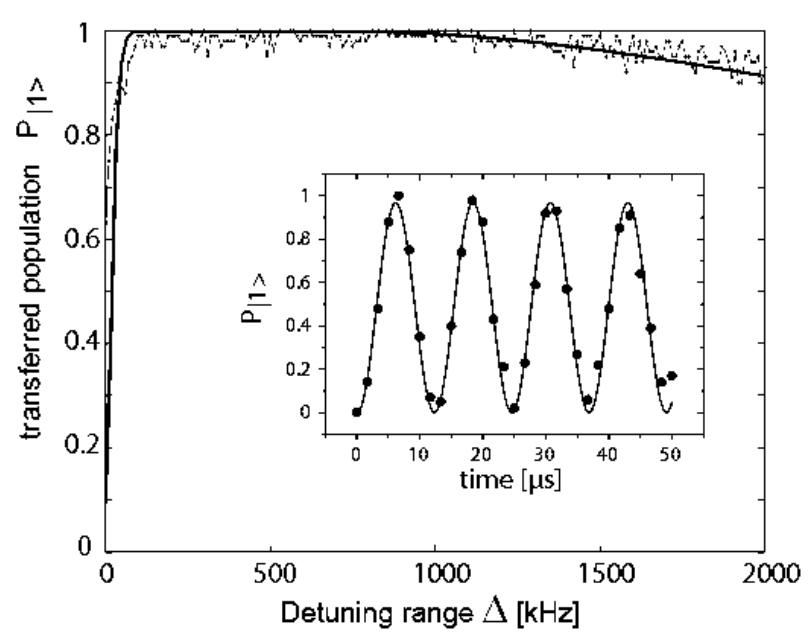

FIG. 2: Adiabatic transfer $|0\rangle \rightarrow|1\rangle$. The excited state population $P_{|1\rangle}$ is plotted for different rates of change of the laser detuning $\delta$ during the pulse, that is for different values of $\Delta \nu / T$ with $T$ fixed at $150 \mu \mathrm{s}$. The transfer works efficiently in the wide range between $\Delta \nu=200 \mathrm{kHz}$ and $\Delta \nu=500 \mathrm{kHz}$, with average efficiency of $99.0(1.0) \%$. The solid line represents the result of an atomic two-level calculation for $\Omega_{R a b i}=$ $512 \mathrm{kHz}$. Inset: The Rabi frequency is directly determined by observing resonant Rabi oscillations. From a sine fit to this data we obtain $\Omega_{R a b i}$ with a fractional error of $0.5 \%$. Thus, all parameters for the simulation are independently fixed, and the solid line is not fitted to the data but results entirely from the model.

Relevant parameters for RAP such as the Rabi frequency and the detuning rate are varied in step (b). Keeping the time $T=150 \mu$ s constant, the range of detuning $\Delta \nu$ is varied. The results are plotted in Fig. 2 The main experimental result is the wide plateau of efficient population transfer from state $|0\rangle$ to state $|1\rangle$. For $\Delta \nu$ ranging from $200 \mathrm{kHz}$ to $500 \mathrm{kHz} 99.0(1.0) \%$ of the population is transferred from the initial state to the target state. Even for the larger range of detunings from $100 \mathrm{kHz}$ to $600 \mathrm{kHz}$, almost perfect transfer of 98.7(1.1)\% is achieved. The two-state simulation predicts a transfer efficiency close to $100 \%$ for the plateau in Fig. 2 Imperfect state preparation and read-out are expected to limit RAP.

It is this robustness, that should make RAP well suited for pulsed Raman ground state cooling: For ground state cooling, a pulse of laser radiation near the red vibrational sideband at $\nu_{\text {laser }}=\nu_{E_{|1\rangle}}-\nu_{E_{|0\rangle}}-\nu_{\text {trap }}$ is applied to a single ion or a linear crystal of ions e.g. in an experimental situation like 25] for ${ }^{9} \mathrm{Be}^{+}$or for ground state cooling of a crystal of ${ }^{43} \mathrm{Ca}^{+}$ions using two Raman beams near $395 \mathrm{~nm}$. Here, the cooling rate suffers from the fact that the sideband Rabi frequency depends on the vibrational quantum number as $\Omega_{n \rightarrow n-1} \propto \sqrt{n-1}$. Thus, with the relevant cooling transition not driven equally strong in case of a thermal distribution of vibrational states, the cooling rate is decreased and even trapping states might occur at certain vibrational quantum states $n$ if the interaction corresponds to an integer $2 \pi$-cycle 22, 23]. Experimentalists have adapted the duration of the Raman pulses to optimize the scheme. RAP cooling, however, completely transfers all vibrational states from $|0, n\rangle \rightarrow|1, n-1\rangle$ such that the subsequent radiative decay closes the cooling cycle. Involuntarily, in conventional pulsed Raman cooling the carrier transition is weakly excited which leads to heating processes. In contrast, the RAP cooling avoids this problem, as the carrier is excited but de-excited again during the adiabatic ramp.

RAP might be used also for the efficient read-out of qubits in systems with ground state qubits, such as hyperfine states of ${ }^{43} \mathrm{Ca}^{+}, \mathrm{F}=4$ to $\mathrm{F}=3$, or the Zeeman levels $S_{1 / 2}, m_{j}= \pm 1$ in ${ }^{40} \mathrm{Ca}^{+}$. In both cases, a Raman transition can be used for coherent qubit manipulation, but detection of the qubit logic state does not work, as both qubit states would scatter photons under excitation on the dipole transitions near $397 \mathrm{~nm}$ and $866 \mathrm{~nm}$. For electron shelving detection one of the qubit levels has to be transferred to the $D_{5 / 2}$ state which then appears dark under excitation on the dipole transitions. We propose for this transfer a RAP pulse to achieve a robust and reliable read-out. The alternative, a resonant $\pi$-pulse, would require a technically demanding laser frequency stabilization and pulse area calibration. For quantum gate operations techniques similar to RAP have been proposed recently [27].

Further applications are expected in cavity QED schemes. Neutral atoms have been used to demonstrate a deterministic single photon emission [26] and similar schemes are currently investigated for the case of trapped ions coupled to an optical cavity. The emission of deterministic photons relying of RAP may improve the feasibility.

In conclusion, we have shown RAP on a single trapped ion. Experimental data and numerical simulation are in good agreement. The experiments show that the $\pi$ transfer pulse is robust and well suited for quantum logic operations and improved optical cooling techniques.

Acknowledgment Ch. W. and Th. H. acknowledge financial support from Science Foundation Ireland under grant number 03/IN3/I397 and the Deutsche Forschungsgemeinschaft. T. K. acknowledges financial support by the FWF. We acknowledge support by the European commission (QGates).

[1] Quantum Computation and Quantum Information, by M. A. Nielsen and I. L. Chuang, Cambridge University Press, (2000).

[2] A. M. Childs and I. L. Chuang, Phys. Rev. A63, 012306 (2000). 
[3] S. Gulde and M. Riebe, G. P. T. Lancaster, C. Becher, J. Eschner, H. Häffner, F. Schmidt-Kaler, I. L. Chuang, R. Blatt, Nature 421, 48 (2003).

[4] C. A. Sackett, D. Kielpinski, B. E. King C. Langer, V. Meyer, C. J. Myatt, M. Rowe, Q. A. Turchette, W. M. Itano, D. J. Wineland, and C. Monroe. 404, 256 (2000).

[5] C. F. Roos, M. Riebe, H. Häffner, W. Hnsel, J. Benhelm, G. P. T. Lancaster, C. Becher, F. Schmidt-Kaler, R. Blatt, Science 304, 1478 (2004).

[6] M. Riebe, H. Häffner, C. F. Roos, W. Hänsel, J. Benhelm, G. P. T. Lancaster, T. W. Körber, C. Becher, F. SchmidtKaler, D. F. V. James, R. Blatt, Nature 429, 734 (2004).

[7] M. D. Barrett, J. Chiaverini, T. Schaetz, J. Britton, W.M. Itano, J.D. Jost, E. Knill, C. Langer, D. Leibfried, R. Ozeri, and D.J. Wineland, Nature 429, 737 (2004).

[8] Ch. Wunderlich and Ch. Balzer, Adv. At. Mol. Opt. Phys. 49, 293 (2003).

[9] H. K. Cummins, G. Llewellyn, J. A. Jones, Phys. Rev. A 67, 042308 (2003).

[10] J. A. Jones, Phys. Rev. A 67, 012317 (2003).

[11] L. M. K. Vandersypen and I. L. Chuang, Rev. Mod. Phys. 76, 1037 (2004).

[12] L. M. K. Vandersypen, M. Steffen, G. Breyta, C. S. Yannoni, M. H. Sherwood, I. L. Chuang, Nature, 414 883, (2001).

[13] I. Cirac and P. Zoller, Phys. Rev. Lett. 74, 4091 (1995).

[14] F. Mintert and Chr. Wunderlich, Phys. Rev. Lett. 87, 257904 (2001); Chr. Wunderlich, in Laser Physics at the Limit (Springer Verlag, Heidelberg-Berlin-New York, 2001), pp. 261; D. Mc Hugh and J. Twamley, Phys. Rev. A 71, 012315 (2005).

[15] P. A. Ivanov, N. V. Vitanov, Phys. Rev. A 71, 063407 (2005).

[16] R. Feynman, F. Vernon, R. Hellwarth, J. App. Phys. 28,
49 (1957).

[17] E. B. Treacy, Phys. Lett 27A, 421 (1968).

[18] K. Bergmann, H. Theuer, and B. W. Shore, Rev. Mod. Phys. 70, 1003 (1998).

[19] B. Broers, H. B. van Linden van den Heuvell, and L. D. Noordam, Phys. Rev. Lett. 69, 2062 (1992)

[20] B. Wanner, R. Grimm, A. Gruber, D. Habs, H.-J. Miesner, J. S. Nielsen, and D. Schwalm Phys. Rev. A 58, 2242 (1998).

[21] F. Schmidt-Kaler, H. Häffner, S. Gulde, M. Riebe, G. P.T. Lancaster, T. Deuschle, C. Becher, W. Hänsel, J. Eschner, C. F. Roos, R. Blatt, Appl. Phys. B: Lasers and Optics 77, 789 (2003).

[22] R. Blatt, J. I. Cirac and P. Zoller, Phys. Rev. A 52, 518 (1995).

[23] G. Morigi, J. Eschner, I.C. Cirac, P. Zoller, Phys. Rev. A 59, 3797 (1999).

[24] H. Dehmelt: Bull. Am. Phys. Soc. 20, 60 (1975).

[25] B. E. King, C. J. Myatt, Q.A. Turchette, D. Leibfried, W. M. Itano, C. Monroe, and D. J. Wineland, Phys. Rev. Lett. 81, 3631 (1998).

[26] M. Hennrich, T. Legero, A. Kuhn, G. Rempe, Journal of Modern Optics, 50(6), 935 (2003).

[27] N. Sangouard, X. Lacour, S. Gurin, H. R. Jauslin, quant-ph/0505163

[28] Due to the double-pass configuration, the modulation of laser frequency and phase is twice the applied rfmodulation. The gaussian chirped laser field waveform is revealed from the rf-waveform by the independently measured AOM transfer function.

[29] Marconi Inc., Signal gen. 2019A

[30] Brimrose Inc.,TEF-270-100 